This item was submitted to Loughborough's Research Repository by the author.

Items in Figshare are protected by copyright, with all rights reserved, unless otherwise indicated.

\title{
Inspection of wood surface waviness defects using the light sectioning method
}

PLEASE CITE THE PUBLISHED VERSION

PUBLISHER

Professional Engineering Publishing / @ IMECHE

VERSION

VoR (Version of Record)

LICENCE

CC BY-NC-ND 4.0

\section{REPOSITORY RECORD}

Yang, D., Michael R. Jackson, and Robert M. Parkin. 2019. "Inspection of Wood Surface Waviness Defects Using the Light Sectioning Method". figshare. https://hdl.handle.net/2134/4722. 
This item was submitted to Loughborough's Institutional Repository (https://dspace.lboro.ac.uk/) by the author and is made available under the following Creative Commons Licence conditions.

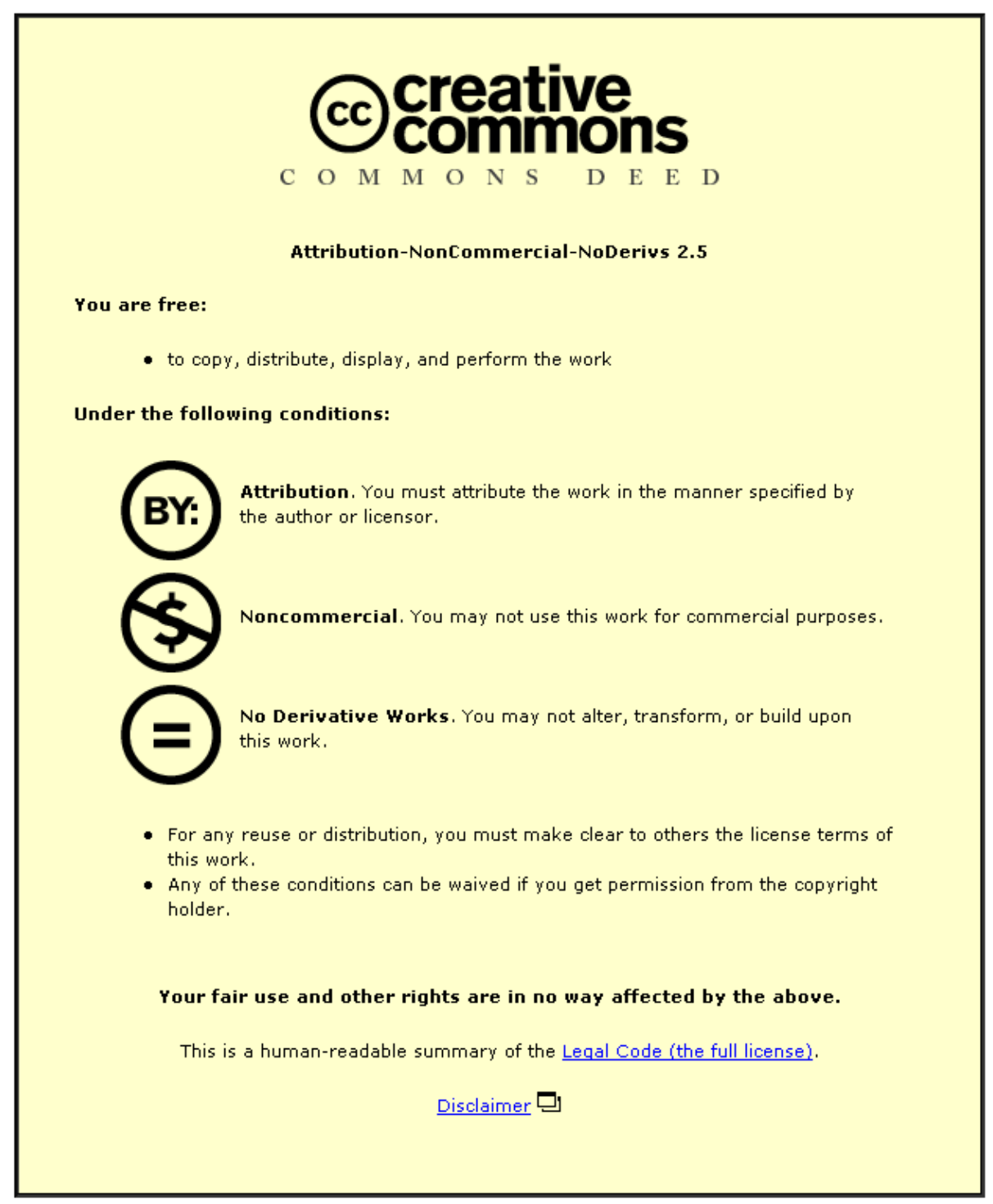

For the full text of this licence, please go to: http://creativecommons.org/licenses/by-nc-nd/2.5/ 


\title{
Inspection of wood surface waviness defects using the light sectioning method
}

D Yang*, M R Jackson, and R M Parkin

Mechatronics Research Group, Loughborough University, Loughborough, UK

The manuscript was received on 30 June 2005 and was accepted after revision for publication on 26 May 2006.

DOI: 10.1243/09596518JSCE175

\begin{abstract}
Surface waviness variations are a major type of defect on planed wood products. A number of methods have been investigated for the inspection of waviness defects on wood surfaces. This paper describes a new implementation of the light sectioning method with the latest structured lighting and machine vision techniques for this purpose. As a reference, a laser profilometer is used. The data from the light sectioning method and from the profilometer are highly correlated.
\end{abstract}

Keywords: wood surface, waviness defect, light sectioning, machine vision, structured lighting

\section{INTRODUCTION}

Planing is a major machining process used in the wood working industry. As illustrated in Fig. 1, the principle of the planing process is very similar to that of the up-milling process in the metal working industry. However, the cutting tool tip velocities and the feed speeds, typically within the range $30-125 \mathrm{~m} / \mathrm{s}$ and $5-120 \mathrm{~m} / \mathrm{min}$ [1] respectively, are much higher than those in the metal working industry. In references [2] and [3], even a feed speed of $200 \mathrm{~m} / \mathrm{min}$ is quoted. Due to the kinematics of the planing process, cutter marks will inevitably be left on the machined surface. However, cutter marks, also termed as waviness in the wood working industry, are actually not defects provided they are small and uniform in width and height. In reality, due to various reasons, such as different rotating radii of cutters on a cutterhead, oscillation of the cutterhead's spindle, and weak structure of machines and so on, the widths and heights of cutter marks on a planed surface are sometimes inconsistent, as shown in Fig. 1. These inconsistencies are known as waviness defects.

\footnotetext{
*Corresponding author: Wolfson School of Mechanical and Manufacturing Engineering, Loughborough University, Holywell Building, Holywell Way, Loughborough LE11 3UZ, UK. email: D.Yang@lboro.ac.uk
}

In this paper, the previous investigations for the inspection of waviness defects on wood surfaces are reviewed first, and at the end of the review a new implementation of the light sectioning method is proposed. After that, the experiment based on the light sectioning method is described, which is followed by data analysis and some discussions.

\section{LITERATURE REVIEW}

For the assessment of waviness defects on wood surfaces, a number of methods have been investigated. In general, these methods are classified into two categories: contact method and non-contact methods.

\subsection{Contact method}

The contact method is always in the form of a stylus tracer. In this method, a stylus is driven across the surface to be measured and surface profiles are produced by recording the vertical movement of the stylus due to the surface height variations and its traverse movement along the surface.

There is a selection of general-purpose stylustracing instruments available on the market, but they are mainly developed for metal surfaces. Peters and Mergen [4], Faust [5], and Jackson [6] developed wood-specific stylus tracing instruments. 


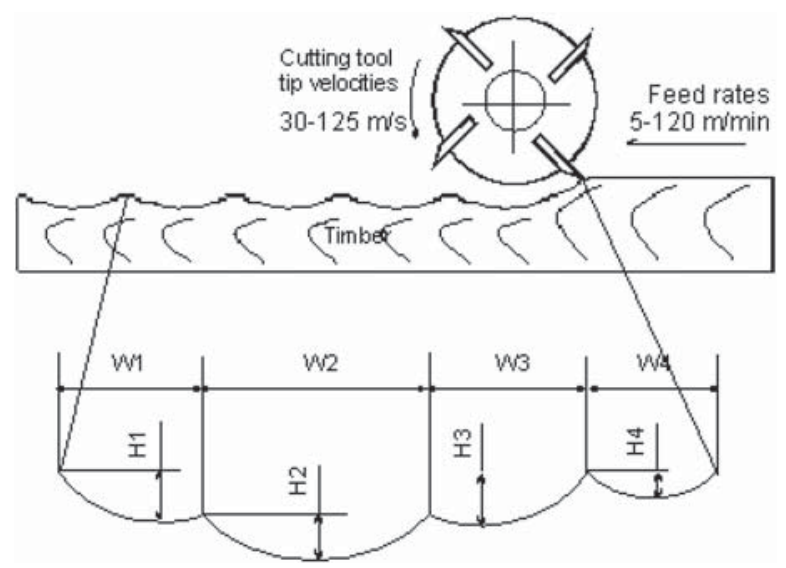

Fig. 1 The schematic of the planing process and the cutter mark parameters

The problems with stylus tracing on wood surfaces were studied in references [6] and [7] and reviewed in reference [8]. One of the major problems is that the stylus, usually made of metal, tends to deform the surface being measured, consequently introducing errors. Another major problem is that the stylustracing technique can only be implemented at a slow speed in order to avoid bouncing (losing contact with the surface). Peters and Mergen [4] and Faust [5] reported $76.2 \mathrm{~mm} / \mathrm{min}$, while Jackson et al. [1] did $0.2 \mathrm{~mm} / \mathrm{s}$. Clearly, this level of measuring speed makes this technique unsuitable for online applications.

\subsection{Non-contact methods}

The most widely investigated non-contact methods for measuring wood surfaces are optical methods. They can be further classified into three methods according to their respective principles:

(a) triangulation sensing;

(b) shadow analysis;

(c) light sectioning.

The triangulation sensing method has been investigated for wood surfaces in references [8] to [12] and for metal surfaces in references [13] to [15]. The principles and some in-depth discussions about this technique can be found in references [16] and [17]. There are some commercial triangulation sensors on the market, such as those from MICROEPSILON (www.micro-epsilon.com) and Keyence (www.keyence.com), although these sensors may be named differently. Potentially, triangulation sensors can measure the surface height with resolution down to $0.1 \mu \mathrm{m}$ or even better $[\mathbf{1 6}, \mathbf{1 7}]$, but they are always quite expensive when high resolution and high accuracy are required. Similar to stylus tracers, triangulation sensors need to scan the surface to produce surface profiles.

There is another technique, known as autofocusing, for measuring profiles of generic surfaces. Because no reference to its applications on wood surfaces has been found, this technique is not introduced here. Later in this paper, however, an autofocusing-based device, referred to as a laser profilometer, is used to produce reference surface profiles, and the technique will be briefly introduced there.

Equipment using triangulation sensors or autofocusing sensors to measure surface quality is sometimes referred to as an optical profilometer. In fact, the optical profilometer is similar to the stylus profilometer in many aspects. The major difference is that the optical profilometer uses a non-contact 'optical stylus', while the stylus profilometer uses a contact stylus.

The shadow analysis method is used to assess surface waviness by analysing the shadows on the surface illuminated by oblique light along the lay, i.e. the feed direction. Hesselbach [3] and Hoffmeister and Grubler [2] used this method to measure cutter mark widths. Maycock [18] used a similar approach to analyse cutter mark variations in the frequency domain. This method was also used in the assessment of wood surface roughness [5]. In general, the shadow analysis method cannot measure surface heights.

The light sectioning method also requires oblique illumination. In this approach, a light stripe is projected from the side of the sample on to the surface to produce a light section. As illustrated in Fig. 2, the light section is actually a wavy line produced by the projected light due to the wavy nature of the surface. Furthermore, as illustrated in Fig. 3, there is a triangular relationship between the height of a cutter mark wave $H$ and the height of its corresponding wave $L$ in the light section, which is $H=L \tan \theta$, or $L=H / \tan \theta$, where $\theta$ is the angle of incidence of the projected light with respect to the surface.

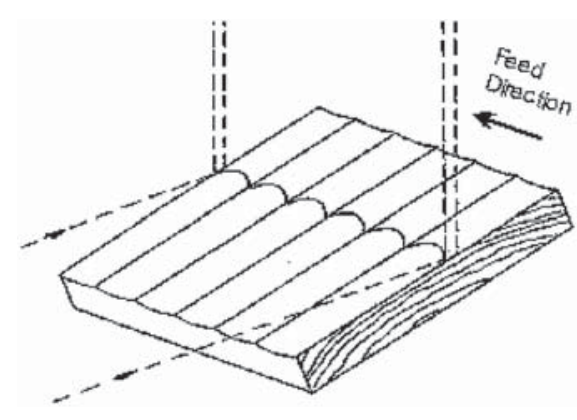

Fig. 2 The principle of light sectioning 


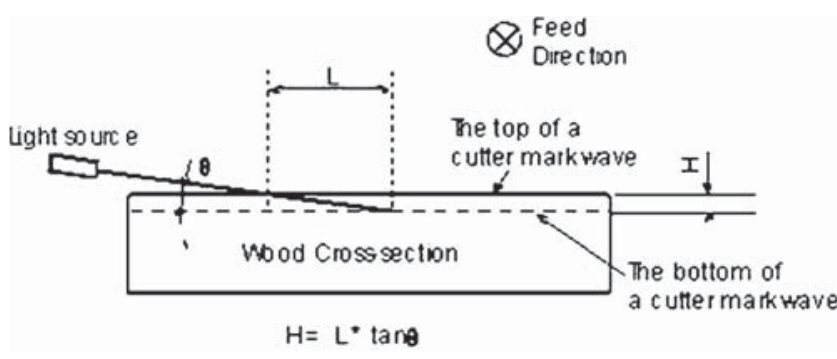

Fig. 3 A cross-section of light sectioning

Therefore, by measuring the wavy line, i.e. the light section, the widths and heights of cutter mark waves on the surface can be calculated.

The light sectioning method is not a new method. The studies on assessing wood surfaces using this approach can be dated back to half a century ago. It is reported in reference [5] that Lutz used this approach to obtain a profile from a wood surface in 1952. In reference [19], it is reported that the Swedish Forest Products Laboratory devised a light sectioning system in 1951 using the same principle, and some sample light sections obtained from the approach are also shown in the reference. Elmendorf and Vaughan also reported a similar technique in 1958 [7]. Since the early 1960s, however, there has not been further research reported. The above-mentioned research may be different from one another in some aspects, but they all used a microscope to magnify the light sections. As a result, the sampling length could not be long. Marian in reference [19] suggested a sampling length of $1 \mathrm{~mm}$; Marian believed that: 'This sampling length is usually too small to be representative of the surface to be tested.' Therefore, it may be reasonably assumed that the light sectioning method could not produce significant results in those days.

However, the light sectioning method has long been successfully applied in some other industrial applications such as inspecting automotive parts $[20,21]$. The difference between those applications and measuring cutter marks on wood surfaces is that the latter may require higher resolution. However, considering the state of the art of the machine vision technologies, the light sectioning method may well provide quantitative analysis of machined wood surface quality.

Compared to the triangulation sensors, this method potentially has a higher measuring rate as it does not need scanning of the surface. Compared to the shadow analysis method, especially the technique developed in references [2] and [3], the light sectioning method is potentially able to measure cutter mark heights.

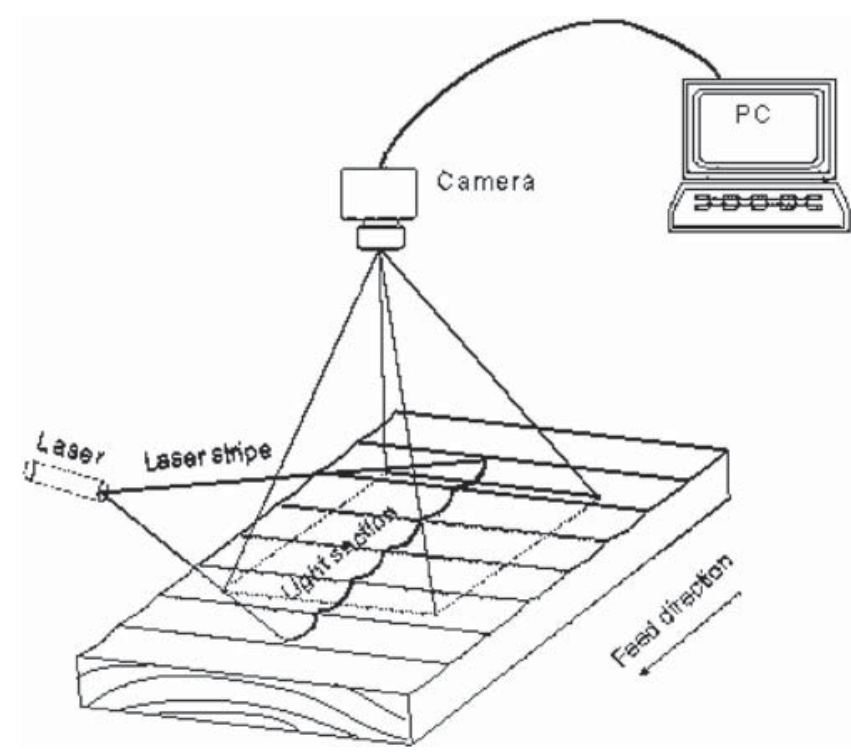

Fig. 4 Test rig set-up

\section{EXPERIMENTAL SET-UP}

According to the principles of the light sectioning method discussed above, a test rig was constructed. As illustrated in Fig. 4, a light source projects a light stripe in a fan from the side of the wood sample. The projected light stripe creates a light section on the sample according to the shape of the surface. The light section, parallel to the feed direction on the surface, is captured by a camera, which is connected to a personal computer (DC).

The light source used in the test rig is a laser line generator. This laser features a patented Powell glass lens designed to generate lines without a Gaussian profile (Refer to http://www.stockeryale.com/i/lasers/ advantages.htm for more information.) Normally, the line generated by an ordinary laser line generator has a Gaussian intensity profile along the length of the line. A comparison of intensity profiles produced by a Gaussian line generator and a non-Gaussian line generator is shown in Fig. 5. Clearly, non-Gaussian line generators will benefit the light sectioning method.

The laser was positioned so that the light came in at a small angle to the sample surface. Due to the triangular relationship shown in Fig. 3, this angle has a predominant influence on $L$, i.e. the heights of the waves in the light section. When the angle $\theta$ is smaller than $6^{\circ}$, the ratio of $L$ to $H$ will be greater than 10 , which is essentially magnifying the heights of the cutter marks. Clearly, the smaller the angle, the greater the magnification. However, if the angle is too small, the light section will appear broken and 
Fig. 5 Comparison of a non-Gaussian line and a Gaussian line

blurred in the image, which makes the subsequent image processing difficult. The image in Fig. 6 is such an example, which was taken with an angle slightly smaller than $1^{\circ}$. Based on the experiments, the angle of incidence was set to between $1.5^{\circ}$ and $6^{\circ}$.

The camera used is a monochrome complementary metal oxide semiconductor (CMOS) camera. The resolution of the camera is up to $1280 \times 1024$, and the CMOS sensor size is $2 / 3$ inch. The lens on the camera is a $100 \mathrm{~mm}$ lens.

The computer used in the test rig is a Celeron ${ }^{\circledR}$ 466 PC with $128 \mathrm{Mb}$ memory. The operating system of the PC is Microsoft Windows 2000 Professional. The image processing software is MATLAB Image Processing Toolbox.

\section{RESULTS AND ANALYSIS}

\subsection{Analysis of a light section}

A number of images were taken from the test rig described above; one of them is shown in Fig. 7. Note that the image in Fig. 7 is only the useful portion of the original image, with those regions only in black removed so that the image is not in the aspect ratio of 5 to 4 . The image was taken in the following conditions:

Object distance: $565 \mathrm{~mm}$

Field of view: $22.5 \times 18 \mathrm{~mm}$

Image resolution: $1280 \times 1024$

Angle of incidence of the laser stripe: $5.7^{\circ}$

Lens $f$-number: 3.5

Exposure time: $72 \mathrm{~ms}$

After applying an edge-finding algorithm to the image in Fig. 7, a profile was obtained as plotted in Fig. 8. Note that the profile in Fig. 8 has had the least squares mean line removed. Clearly, this profile encompasses both the surface waviness and surface roughness.

In order to extract the surface waviness, a low-pass filter has to be applied. There are a number of filter models, such as the sliding average filter, 2CR filter, Gaussian filter, etc. Among them, the Gaussian filter has good roll-off capability and introduces low distortion and phase shift to the data [22]. The Gaussian filter has already been specified as a standard in the metrology industry by the

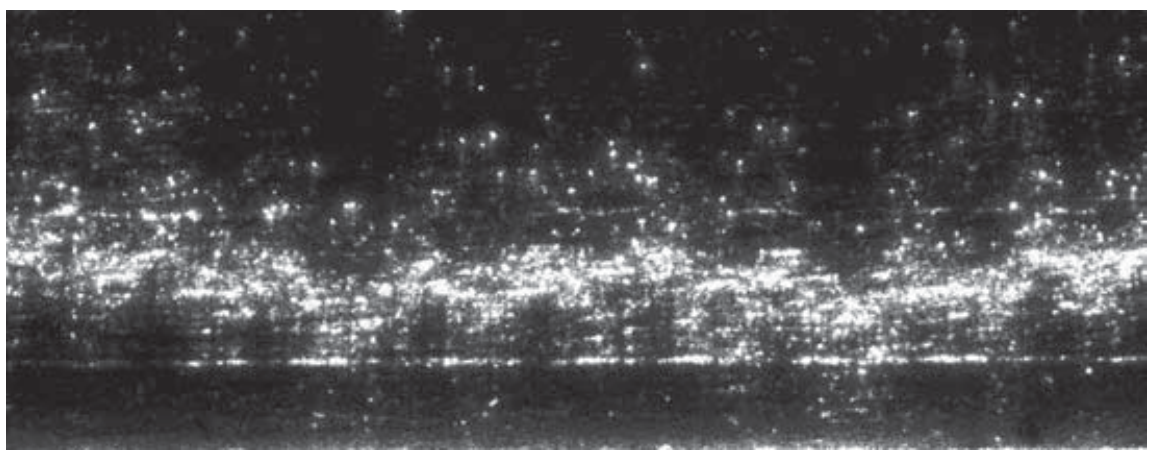

Fig. 6 An example of light sections with too small angles of incidence

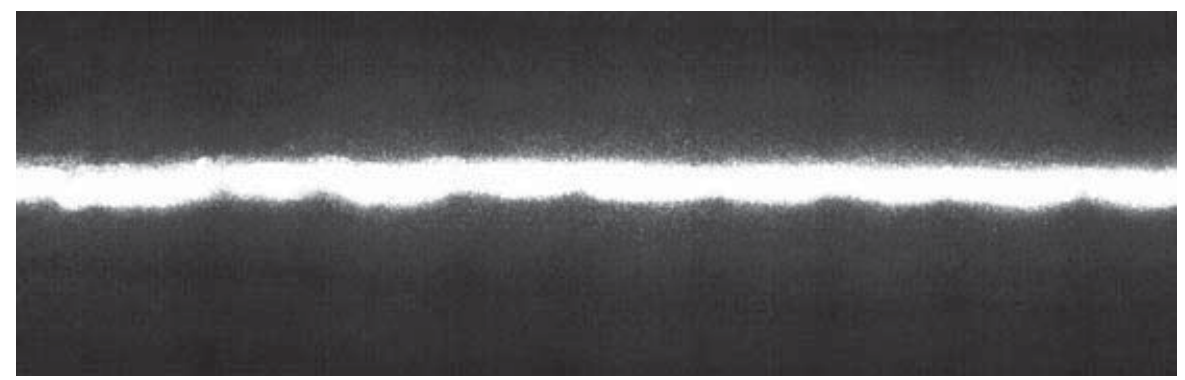

Fig. 7 A light section image 


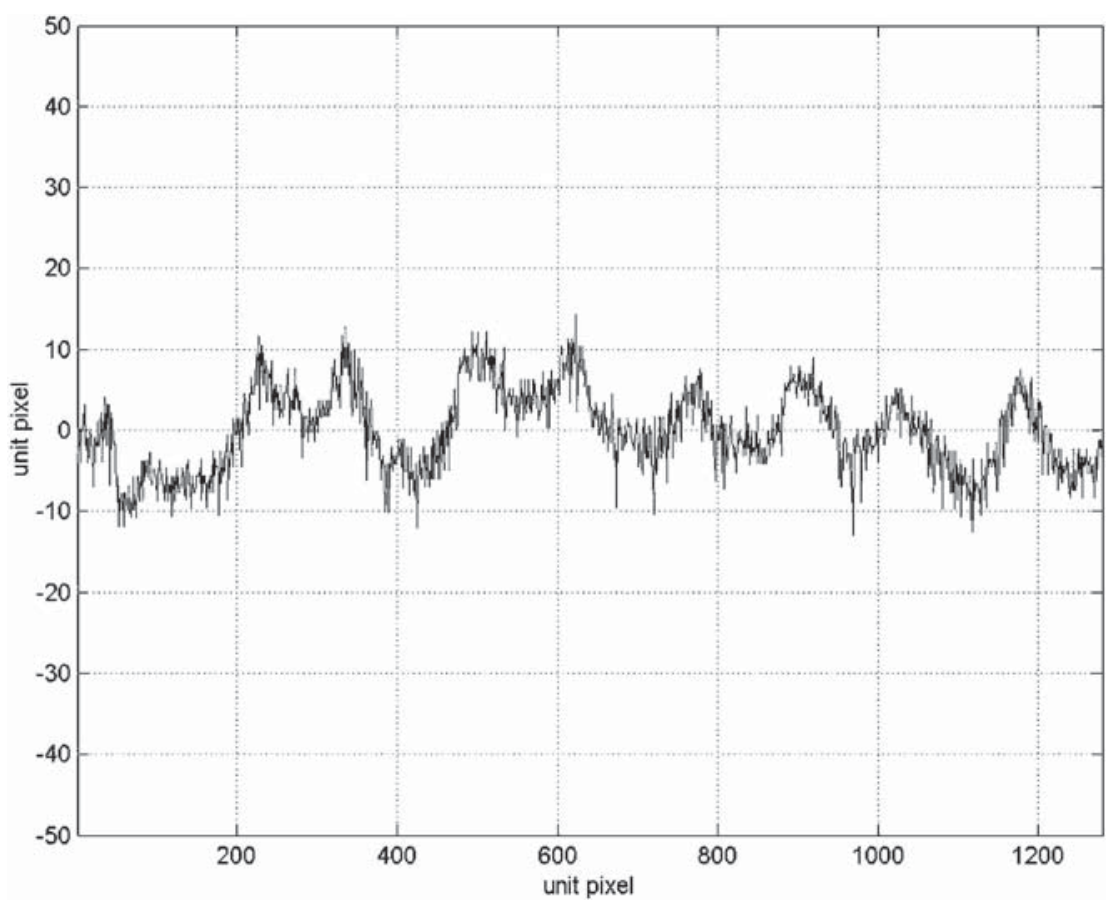

Fig. 8 The profile obtained from the light section shown in Fig. 7

International Standardization Organization (ISO) in ISO 11562 [23]. Essentially, the kernel of the Gaussian filter is a weighting function, which has the shape of a Gaussian density function and is mathematically described by the equation below

$$
g(x)=\frac{1}{\alpha \lambda_{\mathrm{c}}} \exp \left[-\pi\left(\frac{x}{\alpha \lambda_{\mathrm{c}}}\right)^{2}\right]
$$

with $x$ being the distance from the centre of the weighting function, $\lambda_{\mathrm{c}}$ the cut-off wavelength of the filter, and $\alpha$ a constant, defined by

$$
\alpha=\sqrt{\frac{\log 2}{\pi}}=0.4697
$$

Due to the value of $\alpha$, the Gaussian filter is defined to have a transmission of 50 per cent at the cut-off wavelength $\lambda_{\mathrm{c}}$.

A Gaussian filter with a cut-off wavelength of $1.4 \mathrm{~mm}$ is applied to the profile shown in Fig. 8, and the resultant profile is plotted in Fig. 9. In addition, local maximums and local minimums on the filtered profile are marked on the plot.

\subsection{Comparison with a reference}

In order to assess the performance of the light sectioning method, a profilometer was used as a reference. The profilometer is an autofocusing device. The principle of it is to focus a laser spot on the surface being measured and detect the light scattered from a surface with a focus detector; if the focus is lost due to the surface height variations, then the focus detector generates a signal to refocus the lens; while the lens is being refocused, an inductive displacement transducer measures the lens displacement; lens displacements are recorded while the sample moves under the laser so that the surface contour is measured. There is more information on this profilometer available in reference [24].

The vertical resolution of the profilometer is $20 \mathrm{~nm}$ while the horizontal resolution, i.e. the distance the sample moves per step, is selectable, and was set to $25 \mu \mathrm{m}$. Since this profilometer also needs to scan the surface, it is time-consuming to measure a surface with it.

A profile obtained from the profilometer is plotted in Fig. 10. Similarly, this profile has also had the least squares mean line removed. After applying a Gaussian filter with the same cut-off wavelength of $1.4 \mathrm{~mm}$, a filtered profile is obtained and plotted in Fig. 11; the local maximums and local minimums are also marked on the profile. Since the profilometer uses a laser, the profile is referred to as the laser profile in the following text.

In order to compare the light section profile and the laser profile, both profiles are normalized with the following procedures. Re-sample the laser profile with the sampling number of the light section profile, making both profiles have the same scale in the $x$ axis. Convert both profiles using the following 


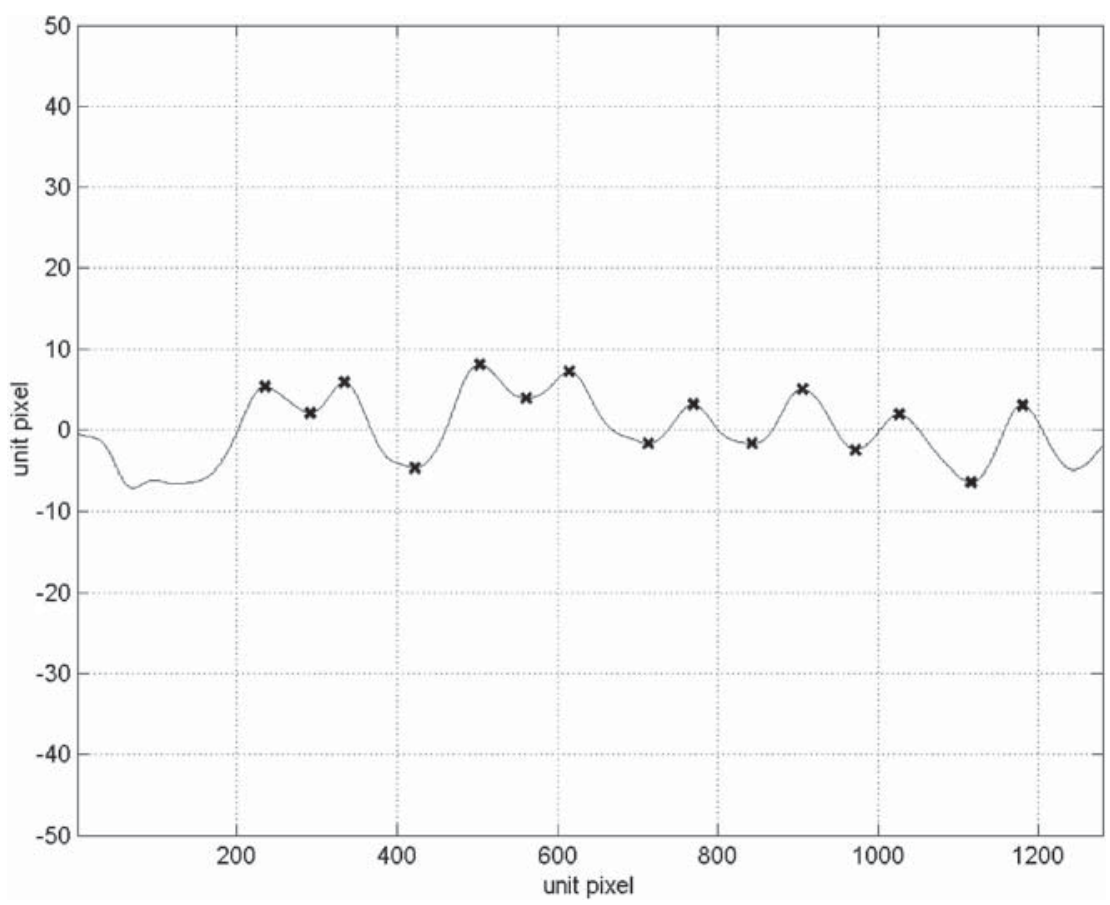

Fig. 9 The filtered light section profile

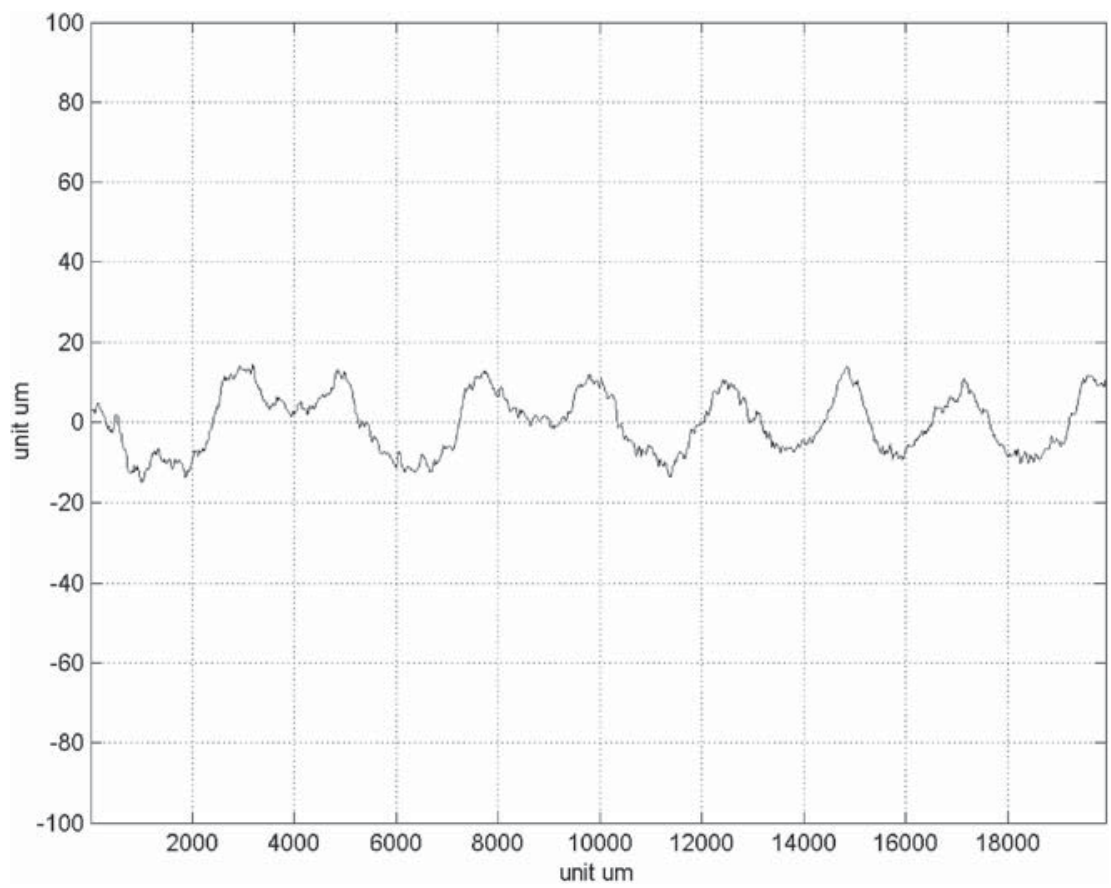

Fig. 10 A profile obtained from the laser profilometer

equation, making both of them have the same scale in the $y$ axis

$$
y=\frac{y_{0}}{\max \left(y_{0}\right)-\min \left(y_{0}\right)}
$$

where $y_{0}$ is the original profile and $y$ is the normalized profile.
After normalization, the light section profile and the laser profile are plotted together in Fig. 12. It can be seen that the light section profile and the laser profile are similar to a large extent. The correlation coefficient of the two profiles is 0.89 , calculated by

$$
\operatorname{corr}(A, B)=\frac{\sum_{m}\left(A_{m}-\bar{A}\right)\left(\bar{B}_{m}-\bar{B}\right)}{\sqrt{\sum_{m}\left(A_{m}-\bar{A}\right)^{2}} \sqrt{\sum_{m}\left(B_{m}-\bar{B}\right)^{2}}}
$$




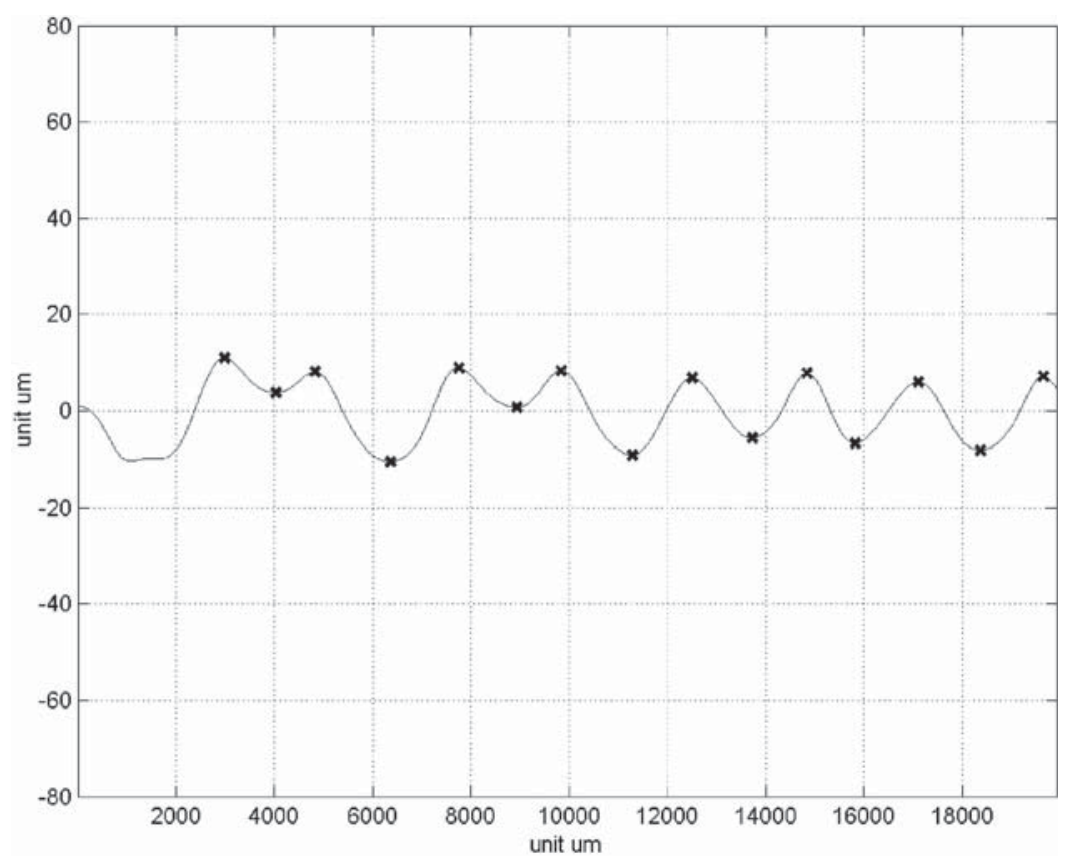

Fig. 11 The filtered laser profile

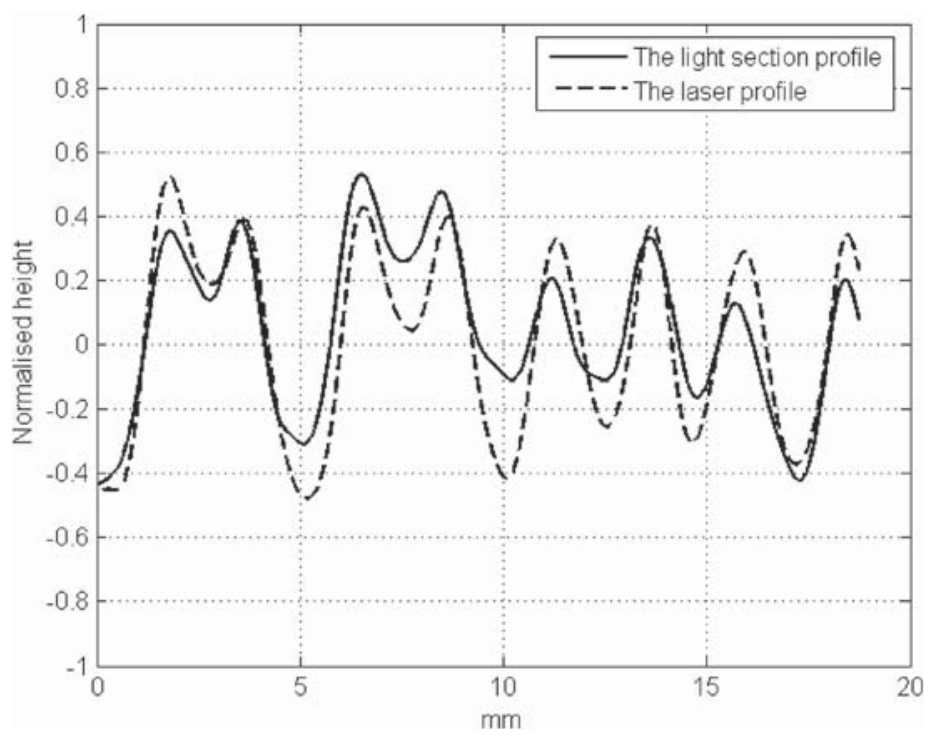

Fig. 12 Comparison of the light section profile and the laser profile

where

$A=$ normalized light section profile and $\bar{A}=$ $\sum_{m} A_{m} / m$

$B=$ normalized laser profile and $\bar{B}=\sum_{m} B_{m} / m$

$m=$ sampling number of $A$ and $B$

\section{DISCUSSION}

The ultimate purpose of this research is to find out a method for online inspection of waviness defects on wood surfaces. As the first step, this paper investigates the feasibility of applying the light sectioning method on static wood samples. Future work will be focused on moving samples. Clearly, moving samples will have blurring problems. However, according to the authors' experience, as long as the exposure time is short enough and a strobe light is used, the blurring will be limited to a small extent. (In a previous project [25], a blur of $1 / 1000$ of the FOV (field of view) was obtained from an object moving at a speed of $3 \mathrm{~m} / \mathrm{s}$.) Besides, deblurring algorithms can be helpful.

In the experimental set-up shown in Fig. 4, the laser stripe is supposed to be projected parallel to the feed direction. In reality, however, it is impossible 
to guarantee this set-up. If the light stripe is not projected parallel to the feed direction, the light section will appear inclined in the image, as illustrated in Fig. 13(b). Figure 13(a) shows a perfectly aligned light section. The vertical lines in Fig. 13 represent the peaks of the cutter marks on the surface. (Here and in the following text, the camera is assumed to be aligned to the surface so that the feed direction of the surface is parallel to one dimension of the FOV of the camera.) Nevertheless, the inclination can be

Top view of the surface and the light section

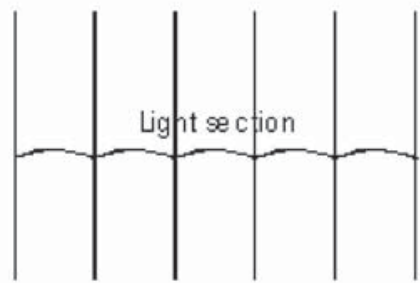

(a) A perfectly a ligned light section

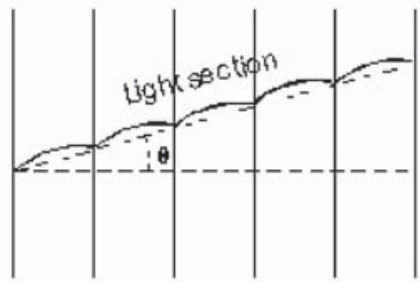

- Angle of misalignment

(b) A misaligned light section

Fig. 13 Misalignment of the light section removed by removal of the least squares mean line of the light section. Considering just this point, the light section profile in Fig. 9 has the least squares mean line removed.

In order to study further the effects of misalignment of light sections, a misaligned light section was simulated. In Fig. 14(a), a cutter mark wave in a light section with $5^{\circ}$ of misalignment is plotted (dashed line) alongside the cutter mark wave with the least squares removed (solid line). In Fig. 14(b), the same cutter mark wave in the same light section with no misalignment is plotted. The simulated cutter mark is $2 \mathrm{~mm}$ wide with a cutting radius of $60 \mathrm{~mm}$, and the angle of incidence of the laser stripe is $5.7^{\circ}$ with respect to the surface. Comparison of the curves in Fig. 14(a) and (b) shows that after removing the least squares mean line, the misaligned light section is almost the same as the perfectly aligned light section. In fact, there is a small difference between the two curves. However, the difference is only in the order of a fraction of a micrometer, which can be ignored compared to the amplitude of the curves. Another simulation with $10^{\circ}$ of misalignment shows the difference is no more than $1 \mu \mathrm{m}$. Further simulations with different cutter mark widths show similar results. Therefore, it is concluded that misalignment of light sections can be acceptably corrected by removing the least squares of the misaligned light section when the angle of misalignment is not too large. Moreover, according to empirical observations, a misalignment larger than $5^{\circ}$ is easy to discern with the eye.

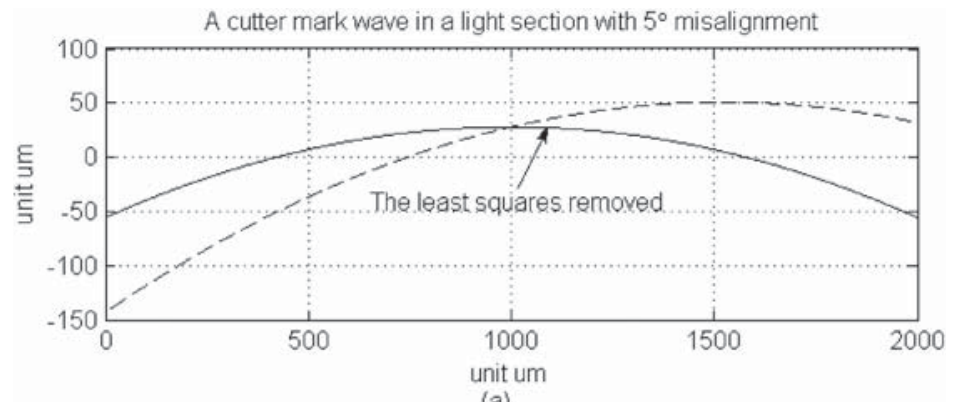

(a)

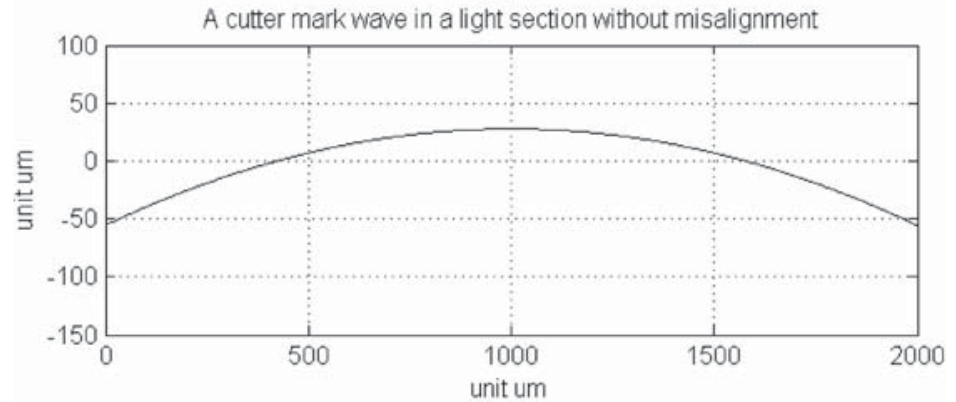

(b)

Fig. 14 Simulated effects of removal of the least squares of a misaligned light section 


\section{CONCLUSIONS}

Waviness defects, in the form of width and height variations of cutter marks on planed wood surfaces, are a major type of surface defect in the wood working industry. A number of methods have been investigated before. This research is based on the light sectioning method, which has not been fully investigated in the wood working industry since it was first studied nearly half a century ago. With the help of the latest machine vision techniques, the light sectioning method has proved in this research to be a feasible solution to the inspection of waviness defects on wood surfaces. More importantly, the light sectioning method can potentially be applied in production lines due to its high data rates and non-contact approach.

\section{ACKNOWLEDGEMENT}

The work reported here was supported by the EPSRC Innovative Manufacturing and Construction Research Centre (IMCRC) at Loughborough University.

\section{REFERENCES}

1 Jackson, M. R., Parkin, R. M., and Brown, N. Waves on wood. Proc. Instn Mech. Engrs, Part B: J. Engineering Manufacture, 2002, 216(B4), 475-497

2 Hoffmeister, H. W. and Grübler, T. In-Prozessmessung der Messerschlagweite gehobetter Massivholzoberflächen Zeitschrift: HOB-Die Holzbearbeitung, 1999, 5, 200-204.

3 Hesselbach, J. Objektive Bestimmung von Jointintervallen durch Messung der Messerschlagsweite, Institute for Machine Tools and Manufacture Technique, Technical University of Braunschweig, 2000.

4 Peters, C. C. and Mergen, A. Measuring wood surface smoothness: a proposed method. Forest Products J., 1971, 21(7), 28-30.

5 Faust, T. D. Real time measurement of veneer surface roughness by image analysis. Forest Products J., 1987, 37(6), 34-40.

6 Jackson, M. R. Some effects of machine characteristics on the surface quality of planed and spindle moulded wooden products. PhD Thesis, Leicester Polytechnic, 1986.

7 Elmendorf, A. and Vaughan, T. W. A survey of methods of measuring smoothness of wood. Forest Products J., 1958, 13(10), 275-282.

8 Yoo, S. M., Dornfeld, D. A., and Lemaster, R. L. Analysis and modeling of laser measurement system performance for wood surface. Trans. ASME, J. Engng for Industry, 1990, 112(2), 69-77.
9 Funck, J., Forrer, J., and Bulter, D. Measuring surface roughness on wood: a comparison of laser scatter and stylus tracing approaches. Soc. PhotoOpt. Instrum. Engrs J., 1992, 1821, 173-184.

10 Lemaster, R. L. and Beall, F. C. The use of an optical profilometer to measure surface roughness in medium density fiberboard. Forest Products J., 1996, 46(11/12), 73-78.

11 Sandak, J., Tanaka, C., and Ohtani, T. Evaluation of surface smoothness by laser displacement sensor. 1: effect of wood species. J. Wood Sci., 2003, 49(4), 305-311.

12 Sandak, J., Tanaka, C., and Ohtani, T. Evaluation of surface smoothness by a laser displacement sensor. II: comparison of lateral effect photodiode and multielement array. J. Wood Sci., 2004, 50(1), 22-27.

13 Costa, M. F. M. Surface inspection by an optical triangulation method. Opt. Engng, 1996, 35(9), 2743-2747.

14 Docchio, F. Design and performance of an optical sensor for the measurement of surface roughness and waviness. Soc. Photo-Opt. Instrum. Engrs J., 1999, 3823, 160-163.

15 Tomassini, P. A novel optical stylus probe for the measurement of surface roughness and waviness. In EUSPEN Topical Conference on Fabrication and metrology in nanotechnology, Denmark, 2001, Vol. 2, pp. 282-289.

16 Pastorius, W. Triangulation sensors: an overview. http://www.lmint.com/Temp/200363017425/ TriangulationSensors.pdf.

17 Pastorius, W. Triangulation sensors: specifications and trade-offs. http://www.lmint.com/Temp/ 200363017426/Specificationsandtrade-offs.pdf.

18 Maycock, K. M. The assessment of surface quality in planed and spindle moulded products. $\mathrm{PhD}$ Thesis, De Montfort University, 1993.

19 Marian, J. E. Surface texture. In Adhesion and adhesives, 1967, pp. 69-86 (Elsevier).

20 Strand, T. C. Optical three-dimensional sensing for machine vision. Opt. Engng, 1985, 24(1), 33-40.

21 Pastorius, W. Machine vision for industrial inspection metrology and guidance. In Fourth Annual Canadian Programmable Control and Automation Technology Conference and Exhibition, 1988, paper 13A2-1/1-5.

22 Exploring surface texture, 2003 (Taylor Hobson Limited).

23 ISO 11562: 1996 Geometric products specifications (GPS) - surface texture: profile method - metrological characteristics of phase correct filters, 1996 (International Standardization Organization, Geneva, Switzerland).

24 Griffiths, B. Manufacturing surface technology: surface integrity and functional performance, 2001 (Penton, London).

25 Brown, N., Jackson, M. R., Parkin, R., and Bamforth, P. E. Machine vision in conjunction with a knowledge-based system for semi-automatic control of the gravure printing process. Proc. Instn Mech. Engrs, Part I: J. Systems and Control Engineering, 2004, 218(I17), 583-593. 


\section{APPENDIX}

\section{Notation}

$g(x) \quad$ Gaussian filter kernel

$H \quad$ cutter mark height (mm)

$L \quad$ light section wave height $(\mathrm{mm})$

W cutter mark width (mm) $\theta \quad$ angle between the incident light and the surface plane

$\lambda_{\mathrm{c}} \quad$ filter cut-off wavelength (mm) 